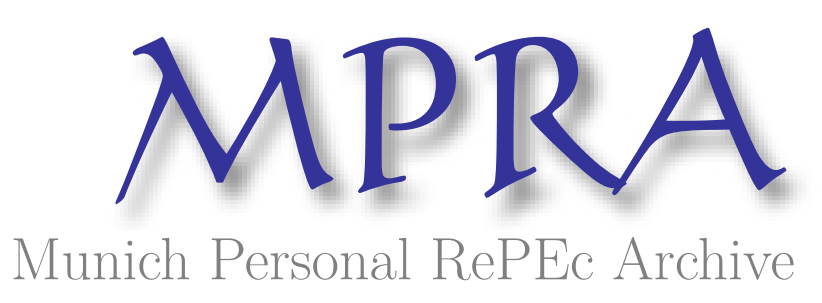

\title{
Why Not Use Robots to Stabilize Stock Markets?
}

Da Silva, Sergio

Federal University of Santa Catarina

30 January 2014

Online at https://mpra.ub.uni-muenchen.de/60567/

MPRA Paper No. 60567, posted 12 Dec 2014 17:02 UTC 


\title{
Why Not Use Robots to Stabilize Stock Markets?
}

\section{Sergio Da Silva*}

Department of Economics, Federal University of Santa Catarina, Brazil

\begin{abstract}
Why not set up some public-service robot traders to counteract the behavior of traders when it snowballs into extreme moves? I show a blueprint of how this can be accomplished taking advantage of the theory of complex systems.
\end{abstract}

Keywords: Robots; Stock Markets; Algorithmic trading; Financial crashes; Flash crash; Mini-flash crashes

My students and I have been suggesting a method for preventing bubbles and crashes in stock markets, other than using monetary policy and financial regulation, which may be ineffective anyway, as we argue [1]. The approach involves setting up a system of "robot traders." By robots we mean "software-based traders," which have been around for years but used for private gain. And they have sometimes been considered as culprits for extreme moves in markets. Some observers blame the first generation of robots for the crash of 1987 as they were the tools for delivering so-called "portfolio insurance." Currently, programs are far more sophisticated and responsible for billions of dollars traded every day. We than asked: Why not set up some publicservice robot traders to counteract the mimetic behavior of traders when it snowballs into extreme moves? Why not create softwarebased trading programs to take contrarian positions at key junctures in the movement of stock markets so as to keep them from diverting to extreme highs and lows? This proposal has received some scant media coverage so far [2].

How can this be accomplished? I will draft an explanation in the follow up, but let me first put that one has to accept as a fact of life that monetary policy and conventional regulation will necessarily fail.

Former Federal Reserve chairman Alan Greenspan believed that bubbles are hard to detect, and that there was little need to respond to bubbles in any case since the Fed can always limit the damage after a bubble pops. Why take the chance of falsely identifying a bubble and slowing the economy unnecessarily when there is little cost to allowing bubbles to run their course? But Great Recessions can happen. And the Fed has evolved a little bit since the pre-bubble days of the Greenspan doctrine. After the subprime bubble, crash, and the Great Recession, Chairman Ben Bernanke acknowledged that the Fed should respond to signs of overheating in stock markets. But he prefers a targeted, regulatory-based approach rather than the use of conventional monetary policy through interest rate increases; the latter is, by the way, favored by Federal Reserve governor Jeremy Stein [3]. I share the concerns of Bernanke on this, and tell you why. Monetary policy is a poor tool crafted for use in a simplistic model world, not the complex financial world we live in. In today's high-tech age, one naturally assumes that policymakers are using sophisticated quantitative computer models to guide monetary policy, but they are not. The best models they have are of two types [4]. The first is econometric: empirical statistical models that are fitted to past data. These successfully forecast a few quarters ahead as long as things do not change, but fail in the face of big changes. The second type is known as DSGE (Dynamic Stochastic General Equilibrium) models. These models by their very nature rule out crises, and thus are also a poor tool to tackle big changes. But there are alternatives that suit the complexity of the matter; these are the class of "agent-based models." So to summarize, monetary policy is a tool not designed to our complex financial world. One has to move on to an upper level and embrace the superior approach of agent-based models. Unfortunately, mainstream economists and policymakers for that matter are not prepared-and some are even hostile-to acknowledge that financial markets are complex systems. This sounds "too specialized" and "too scientific" for the average economist's mindset.

Conventional financial regulation is also hopeless. Experience from the recent crisis suggests it is in the nature of markets that they will tend to innovate around regulations, and the nature of risk taking will inevitably keep changing as financial systems get more sophisticated [5]. Regulatory frameworks have to adapt to circumstances that are changing too fast for regulation to succeed, and the robots have something to do with this. The recent episodes of "flash crashes" provide an example. Thanks to the flash crash of May 6th when the Dow Jones Industrial Average plunged by nearly 1,000 points in a matter of minutes, the merits of high-frequency trading are under scrutiny by regulators, in particular the Securities and Exchange Commission. The flash crash report [6] identified that ...on May 6, when markets were already under stress, the sell algorithm chosen by the large trader to only target trading volume, and neither price nor time, executed the sell program extremely rapidly in just 20 minutes. At a later date, the large fundamental trader executed trades over the course of more than 6 hours to offset the net short position accumulated on May 6 .

The report then concluded that...one key lesson is that under stressed market conditions, the automated execution of a large sell order can trigger extreme price movements, especially if the automated execution algorithm does not take prices into account.

The Securities and Exchange Commission then suggested a marketwide system of "circuit breakers," which would require all exchanges to stop or slow down trading for a few minutes if the market experiences a certain rate of decline. I call your attention for the fact that the SEC does not aim to act pre-emptively, but only to react in the aftermath. It is no surprise then that "mini-flash crashes" in individual stocks have been left unchecked since the flash crash of the DJIA. Stocks that experienced rapidly plunges then rebounds on particular days in 2011 and 2012 were Abott Labs, Apple, Cisco Systems, City Group, Core Molding, Enstar, Jazz Pharmaceuticals, Micron, Progress Energy,

*Corresponding author: Sergio Da Silva, Department of Economics, Federal University of Santa Catarina, Brazil, Tel: +554837219901; E-mail: professorsergiodasilva@gmail.com

Received December 11, 2013; Accepted February 10, 2014; Published February 12, 2014

Citation: Da Silva S (2014) Why Not Use Robots to Stabilize Stock Markets? J Stock Forex Trad 3: 120. doi:10.4172/2168-9458.1000120

Copyright: ( 2014 Da Silva S. This is an open-access article distributed under the terms of the Creative Commons Attribution License, which permits unrestricted use, distribution, and reproduction in any medium, provided the original author and source are credited. 
Pfeizer, Pall Corporation, RLJ Equity Partners, Thermo Fischer Co., and Washington Post. Stock exchanges do not publicly release data about these mini crashes, but most active traders say there are currently at least a dozen a day [7]. In summary, apparently financial regulation in its current format is not working.

Bak and Paczuski remarkably observed [8] that in complex systems, large, catastrophic events occur as a consequence of the same dynamics that produces small, ordinary events. This statement runs counter to the usual way of thinking about large events. But large dynamic systems naturally evolve, or self organize, into a highly interactive, critical state where a minor perturbation may lead to events of all sizes. Such actual events cannot be predicted, but the statistical distribution of these events is predictable. As stock markets are viewed as complex systems, the trigger of a crash cannot be synonymous with its cause. A confluence of factors rather than a particular trigger is the proper explanation. Take the trigger of the flash crash, which the SEC identified as the sell order of the mutualfund group Waddel \& Reed, which started to sell $\$ 4.1$ billion of "E-Mini" futures contracts through robot trading. According to the SEC, this ignited the crisis because the markets were already "under stress." I have to add for the sake of precision that the DJIA had to be in a critical state on May 6 for such a sell order to trigger the flash crash. Otherwise, the crisis would ever get started.

In fact, the flash crash of the DJIA shows the footprints of a complex system at work; I have witnessed this in a study with another couple of students [9]. And with a colleague we went further [10]. Thanks to increasing high-frequency trading, correlations previously only seen across hours or days in trading time-series are now possibly showing up in timescales of minutes or seconds [11]. As it happens, the buildup of correlations brought by high-frequency trading may shape the nature of flash crashes and these may emerge as "log-periodic phenomena." This means that after a critical time a crash may suddenly occur without any early warning signs.

Despite the fact that financial regulation cannot succeed using the currently widely used conventional tools, in theory one can still rely on the control theory of self organized systems [8]. This approach has been applied to a number of complex systems, including the attempts to influence the group behavior of cockroach aggregation in shelters. Engineers have devised autonomous cockroach-robots and relied on self organization as the main coordination mechanism. The controller of individual robots was designed using reactive, behaviorbased techniques. Socially integrated autonomous robots, perceived as conspecifics by the group of cockroaches and acting as interactive decoys, were able to control their self-organized group choices of shelter. Inspired by this, my students and I then suggested the use of socially integrated robot traders in stock markets to function as an anti-bubble decoy [1]. We borrowed from such models of cockroaches finding a shelter but also from those of information transfer in fish shoals, and applied their modeling principles to the stock market. Our basic framework was a type of agent-based model named "stochastic cellular automata model." The model was able to generate an emergent stock price dynamics as a result of the interaction between traders. We then replicated the characteristics of actual stock markets with their price dynamics of highs and lows. In the presence of extreme events, these cannot be accommodated with a Gaussian probability distribution.
Variances in the real financial world are too high for Gaussian standards. But we were lucky enough to realize that after introducing socially integrated contrarian robots, the stock price dynamics could be controlled, so as to make the market more Gaussian. This plainly means the bubbles and crashes disappeared. So we think this blueprint for market stability, if correctly engineered, may offer a credible alternative to monetary policy and current financial regulation. And while monetary policy and standard financial regulation are conspicuous, an extra advantage of contrarian algorithms is their crypticness.

We are not selling the final recipe for preventing financial crashes; rather, we are suggesting the ingredients and the type of cake. How to pay for the costs of the contrarian robot-trading system still needs refining. One implicit implication of our analysis [1] was that 20 percent of the total market turnover would give the necessary resources, because we found that 20 percent of robots were enough for stabilizing. Obviously, this is too expensive for the taxpayer. And this will render the approach unfeasible in practice, unless the robots are adjusted to make money as well, in which case the contrarian algorithms will be self-financing. An extra difficulty is that the human investors will likely react to the counter-measures. To circumvent this barrier, research will have to appeal to the insights provided by biological studies of evolutionary arms race. The regulatory robots would take advantage for acting first, but should be prepared to react differently in light of possible disclosures by investors who decode the running algorithm and attempt to exploit it. Anyway, any artificial-market model like ours can capture some key characteristics of actual markets but inevitably may neglect others, thanks to the very nature of modeling. Thus, the line of research launched by our approach still needs further model distilling. And as more and more robust estimation are produced we will end up with the ultimate recipe for practical use.

\section{References}

1. Suhadolnik N, Galimberti J, Da Silva S (2010) Robot traders can prevent extreme events in complex stock markets. Physica A 389: 5182-5192.

2. MacDonald L (2010) Preventing bubbles with robot traders. Canadian Business, September 13

3. Thoma M (2013) Can the Fed burst the next bubble before it's too late? The Fiscal Times, March 12.

4. Farmer J D, Foley D (2009) The economy needs agent-based modelling Nature 460: 685-686.

5. Edey M (2009) The global financial crisis and its effects. Economic Papers 28 186-195.

6. Staff of the US (2010) Commodity Futures Trading Commission and the U.S Securities and Exchange Commission. Findings regarding the market events of May 6, 2010, September 30

7. Farrell M (2013) Mini flash crashes: A dozen a day. CNN Money, March 20.

8. Bak P, Paczuski M (1995) Complexity, contingency, and criticality. Proc Natl Acad Sci USA 92: 6689-6696.

9. Mazzeu J, Otuki T, Da Silva S (2011) The canonical econophysics approach to the flash crash of May 6, 2010. Applied Mathematical Sciences 5: 1373-1389.

10. Matsushita R, Da Silva S (2011) A log-periodic fit for the flash crash of May 6 , 2010. Economics Bulletin 31: 1772-1779.

11. Smith RD (2010) Is high-frequency trading inducing changes in marke microstructure and dynamics? ArXiv Quantitative Finance Paper 1006.5490.

Citation: Da Silva S (2014) Why Not Use Robots to Stabilize Stock Markets? Stock Forex Trad 3: 120. doi:10.4172/2168-9458.1000120
This article was originally published in a special issue, Economics, Finance and Public Policy handled by Editor(s). Dr. Jimmy Teng, University of Nottingham, Malaysia 